\title{
Anamnestic Immune Response Eight Years After Immunization of Primates with a Multivalent HIV-1 GP120 Variable Peptide Vaccine
}

\author{
${ }^{1}$ Rebecca Rivera, ${ }^{1}$ Kyung Hee Kang, ${ }^{1}$ David E. Anderson, \\ ${ }^{1}$ Juan P. Marquez, ${ }^{2}$ Murray B. Gardner, ${ }^{1} \mathrm{Xia}$ Li and ${ }^{1} \mathrm{Jose}$ V. Torres \\ ${ }^{1}$ Department of Medical Microbiology and Immunology, \\ ${ }^{2}$ Department of Medical Pathology and Laboratory Medicine and Center of Comparative Medicine, \\ School of Medicine, University of California, Davis, California, USA
}

Received 2013-01-06, Revised 2013-04-10; Accepted 2013-05-13

\begin{abstract}
Successful development of an effective HIV vaccine hasn't occurred yet partly as a consequence of the antigenic variation deployed by HIV-1 to escape the immune system. Our laboratory is dedicated to develop a single peptide synthesis approach to create multivalent peptides representing hypervariable epitopes of the gp120 envelope glycoprotein of HIV-1. Our previous study showed that our HIV HECs are potent immunogens that activate both humoral and cellular arms of the acquired immune response and that these responses are broadly reactive, recognizing epitopes from divergent strains of HIV-1. To detect the long term duration of memory response induced by HIV HECs, two rhesus macaques were immunized at weeks 0 and 8 and euthanized two weeks after a third immunization at week 393 (more than 8 years later). Antibody response to individual components of HIV HEC immunogens and HIV HEC-induced cross-reactive antibody response were determined by an Enzyme-Linked Immunosorbent Assay (ELISA). The antibody titer to individual HIV HEC components and a mixture of the five peptides was greater than 1:5000 dilution. Antibodies from HIV HEC-immunized macaques recognized HIV HEC analogs representing the monovalent epitopes of five variable regions of gp 120 from subtype B HIV-1 MN, HIV-1 RF and HIV-1 SF2 isolates with an antibody titer greater than 1: 500 dilution. Moreover, lymphocytes from lymph nodes of HIV HEC-immunized macaques showed $\mathrm{T}$ cell proliferative responses specific to HIV HEC individual components and to the five HIV HEC peptides combined. Our results clearly show that in these two macaques, HIV HECs induced strong, long-lasting anamnestic immune responses 8 years after immunization.
\end{abstract}

Keywords: HIV-1, Hypervariable Epitope Constructs (HECs), Long Term Immunological Memory

\section{INTRODUCTION}

For over two decades, numerous research groups have tried to develop HIV vaccines (Gallo, 2005; Levy, 2006). However, no currently available vaccines confer a protective immune response against HIV infection or delay AIDS progression of infected patients (Ndung'u and Weiss, 2012; Saunders et al., 2012). All candidate vaccines that have undergone clinical trials in humans have resulted in lack of significant protection against HIV infection. Many HIV vaccine trials designed to elicit a protective immune response against the envelope glycoprotein gp120 have been hampered because of conformational masking of receptor-binding sites (Kwong et al., 2002), epitope occlusion and posttranslational modification by glycosylation (Overbaugh and Rudensey, 1992; Wyatt et al., 1998) and the existence of immunosuppressor determinants

Corresponding Author: Rebecca Rivera, Department of Medical Microbiology and Immunology, School of Medicine, University of California, Davis, California, USA 
(Shan et al., 2007). In spite of these obstacles, development of an effective HIV vaccine may still be possible. However, to achieve this goal more knowledge of the in vivo immune response at the initial site of infection and the systemic immune responses that control HIV replication and disease progression is needed.

One of the major problems associated with the development of retroviral vaccines is to overcome the antigenic variability of multiple epitopes attributed to error-prone reverse transcriptase and RNA polymerase. Our laboratory has developed an approach to prepare peptides in a single synthesis that represent the cumulative epitope variability observed in vivo in HIV-1 infected subjects. HIV Hypervariable Epitope Constructs (HECs) are synthetic peptide mixtures that contain multiple variants of a given epitope, based on five variable regions of the envelope glycoprotein of HIV-1 (gp120).

Previously, we examined the immunogenicity of HIV HECs in animal studies, showing that the HIV HECs are highly immunogenic, eliciting both humoral and cellular immune responses. However, because of the high cost of nonhuman primate maintenance, we were not able to determine the long-term duration of the immune response elicited by the HIV HECs. In the present study, anamnestic antibody and lymphocyte proliferative responses assessed eight years after HIV HEC vaccination of non-human primates are briefly reported.

\section{MATERIAL AND METHODS}

\subsection{HIV HEC Design and Construction}

The method for synthesizing and designing the HEC peptides based on antigenic variable regions of viral proteins has been described (Anderson et al., 1994; 2005; Carlos et al., 2000; Reddy et al., 2004; Azizi et al., 2008). Briefly, amino acid sequence data corresponding to five hypervariable immunogenic regions of HIV-1 gp120 were obtained from Los Alamos Human Retroviruses and AIDS database. The protein sequences from all available isolates were aligned and the possible amino acids for each position along the variable epitopes were determined. HIV HECs were synthesized by 9-fluorenylmethyloxycarbonyl (Fmoc) chemistry, a solid-phase peptide synthesis method, utilizing high capacity $(0.7 \mathrm{mmol} / \mathrm{g}) \quad$ Knorr resin (Advanced Chemtech).

\subsection{Immunization of Rhesus Macaques with HIV HECs}

Animals in this study were housed at the California National Primate Research Center (Davis, CA) according to the regulations of the Association for Assessment and Accreditation of Laboratory Animal Care International standards and the institutional Animal Care and Use Committee of the University of California, Davis. Two adult male rhesus macaques (mac 25598 and mac 25705), free of Simian Immunodeficiency Virus (SIV), Simian Retrovirus (SRV) and Simian T-cell Leukemia Virus (STLV), were immunized intramuscularly (i.m.) with 500 $\mu \mathrm{g}$ of an equimolar mixture of five HIV HEC peptides resuspended in an equal volume of Phosphate-Buffered Saline (PBS) and Montanide ISA51 adjuvant (Seppic Inc). Rhesus macaques were immunized two times at weeks 0 and 8 and euthanized two weeks after a third immunization at week 393 (more than 8 years later).

\subsection{Detection of Antibody Response by ELISA}

Antibody response to individual components of HIV HEC immunogens and HIV HEC-induced cross-reactive antibody response were determined by an EnzymeLinked Immunosorbent Assay (ELISA). The method of performing ELISA to detect HEC-specific antibody responses has been described (Carlos et al., 2000; Anderson et al., 2005; Kang et al., 2010). Plasma obtained prior to immunization was used as negative control.

\subsection{Lymphocyte Proliferative Response}

Lymph nodes were obtained on the day of euthanization and lymphocytes were isolated by FicollPaque density gradient centrifugation. $1 \times 10^{5}$ cells per well in 96 well round-bottom plates were stimulated with each individual HIV HEC or with a combination of all five HIV HECs in a final concentration of 10 $\mu \mathrm{g} / \mathrm{mL}$ in RPMI 1640 medium containing $10 \%$ Fetal Bovine Serum (FBS), L-glutamine and antibiotics. Following 6 days of culture, $1 \mu \mathrm{Ci}$ of $\left[{ }^{3} \mathrm{H}\right]$ thymidine was added to each well and cultured for $16-18 \mathrm{~h}$ prior to harvest. $\left[{ }^{3} \mathrm{H}\right]$ thymidine incorporation was determined by measuring radioactivity in an LKB Wallac liquid scintillation counter. The results were expressed as Count Per Minutes (CPM).

\subsection{Statistical Analysis}

The significance of differences between groups was determined using a paired Student $t$ test (with a confidence level of 95\%) with Graph Pad Prism software, version 5.01. Two-tailed $P$ values of $<0.05$ were considered statistically significant.

\section{RESULTS AND DISSCUSSION}

Our laboratory has developed a novel method to prepare synthetic peptide immunogens based on antigenic epitopes of variable regions of viral proteins. 


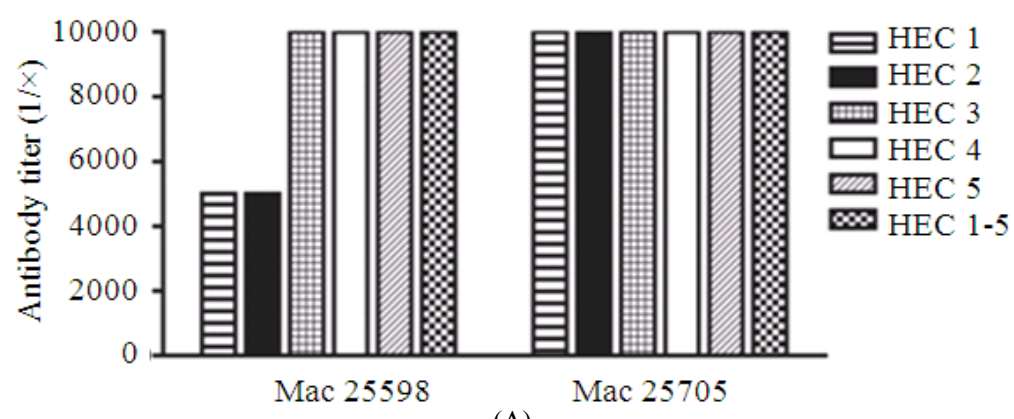

(A)

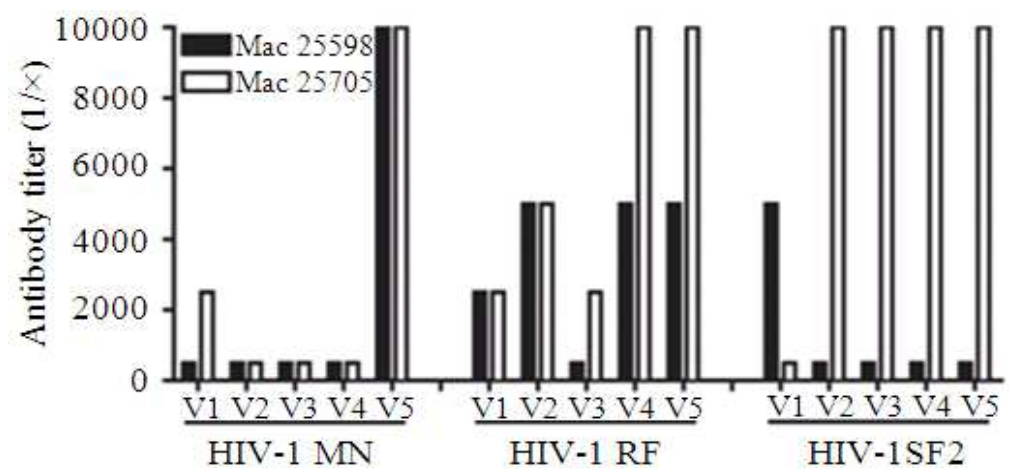

(B)

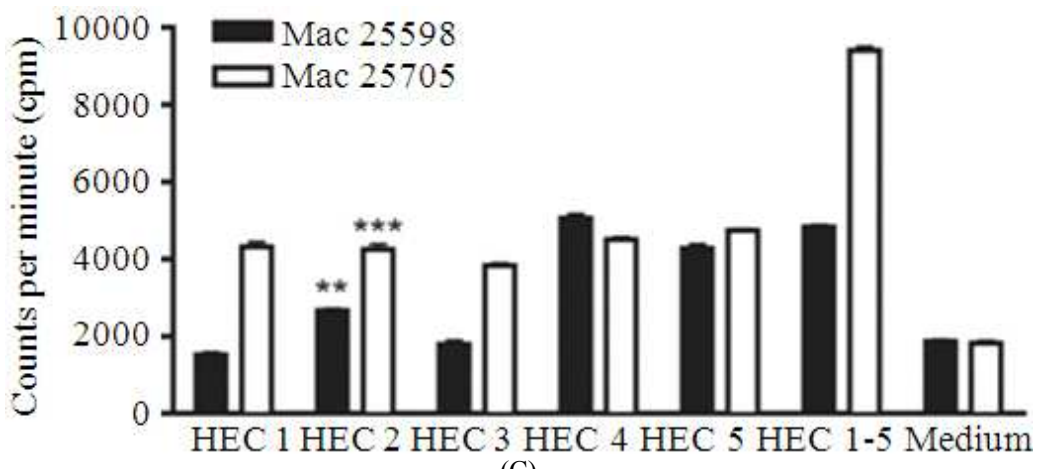

(C)

Fig. 1. HIV HEC-specific antibody response

Sequences of HIV HECs representing the five variable regions of gp120 envelope glycoprotein of HIV-1 are shown in Table 1. Sequences of analogs, single sequence peptides representing the variable regions of the gp120 envelope glycoprotein of subtype B HIV-1 MN, HIV-1 RF and HIV-1 SF2 isolates are previously described (Carlos et al., 2000).

In a previous study, we evaluated the immunogenicity of HIV HECs in rhesus macaques. Two male rhesus macaques (mac 25598 and mac 25705) were immunized intramuscularly with HIV
HECs two times in a two-month interval (week 0 and 8 ) and both of them developed strong, broadly reactive binding antibodies and $\mathrm{T}$ cell proliferative responses specific to the HIV gp120 sequences represented (Carlos et al., 2000).

To further understand the duration of memory response elicited by the HIV HECs, these two macaques received a boosting immunization with the same amount of HIV HEC peptide mixture 8 years (week 393) after the second immunization and the HIV HEC-specific memory response was determined. 
Rebecca Rivera et al. / American Journal of Immunology 9 (1): 30-35, 2013

Table 1. HIV HECs based on the V1-V5 variable regions of gp 120 envelope glycoprotein of HIV-1

\begin{tabular}{|c|c|c|c|}
\hline Construct and region & HIV HEC Sequence & HLA alleles & Variants \\
\hline HEC 1 & CTDLKNDTNTTNSTSGRMMMEK & DQA1*0501; & \\
\hline V1 (aa 130-151) & --- N---- A-------T-N--E------- & $\begin{array}{l}\text { DRB } 1 * 0301, * 0401, * 1302, * 1501 ; \\
\text { DRB3 } * 0202\end{array}$ & 32 \\
\hline HEC 2 & FYKLDIVPIDNTTTSYRRLISC & DPA $1 * 0102,{ }^{*} 0201$ & \\
\hline V2 (aa 161-181) & --- -B----------- DSN--------- & $\begin{array}{l}\text { DQA } 1 * 0501 ; \\
\text { DRB } 1 * 0101, * 0301, * 1104,{ }^{*} 1301, * 1302 \\
\text { DRB3 } * 0202\end{array}$ & 16 \\
\hline HEC 3 & NNNTRKSIRIGPGRAFYTTGDIGNIR & DPA $1 * 0102$ & \\
\hline $\begin{array}{l}\text { V3 (aa } 305-331) \\
\text { HEC } 4\end{array}$ & ----------- R-H------ Q--------- E--D--- & $\begin{array}{l}\text { DQA } 1 * 0501 ; \text { DRB } 1 * 0101, * 0301, * 1501 ; \\
\text { DPA1*0102; }\end{array}$ & 32 \\
\hline V4 (aa 390-413) & $\begin{array}{l}\text { FNSTWFNSTWSTEGSNNTEGSDT } \\
\text { - }\end{array}$ & $\begin{array}{l}\text { DQA } 1 * 0501 ; \text { DRB } 1 * 0402, * 0901, * 1301 ; \\
\text { DRB3*0202 }\end{array}$ & 4 \\
\hline $\begin{array}{l}\text { HEC } 5 \\
\text { V5 (aa 454-475) }\end{array}$ & $\begin{array}{l}\text { LTRDGGNNNNESEIFRPGGGD } \\
\text {--------- T-T------ }\end{array}$ & $\begin{array}{l}\text { DQA } 1 * 0501 \\
\text { DRB } 1 * 0101, * 0301, * 1302, * 1501 \\
\text { DRB3 } * 0202\end{array}$ & 4 \\
\hline
\end{tabular}

Positions in which two amino acids are added at a coupling step are highlighted in bold. Dashes indicate identical amino acid sequence in which a single amino is added. Each HEC contains 4, 16 or 32 variants as indicated. The locations of the V1-V5 epitopes are indicated for a reference strain of virus (HIV-1 MN). Representative sequences from each HEC were screened for the presence of epitopes predicted to bind to any HLA class II epitopes with defined-binding motifs. Those HLA alleles predicted to bind to an epitope contained within the HEC are indicated. Predictions were made with HLA Ligand and Motif Database funded by NIH.

Plasma was obtained from HIV HEC-immunized macaques 8 days after the boost immunization and plasma obtained prior to boosting was used as negative control to determine the basal level of antibody response. HIV HEC-specific antibody response was determined by ELISA. Antibody response was considered positive when the OD value from plasma of boosted macaques was at least twice as that of pre-immunization. The endpoint antibody titer was defined by the highest dilution in which antibody response was considered positive. The antibody titer to individual HIV HEC components and a mixture of the five peptides was greater than 1:5000 dilution (Fig. 1A). These results suggest that both macaques still have HIV HEC-specific memory and or plasma B cells capable of producing antibodies that recognize each HIV HEC peptide with strong affinity 8 years after immunization.

Two weeks after a booster immunization, macaques were euthanized and the cross-reactivity of HIV HEC-induced antibody response and HIV HECspecific lymphoproliferative response were assessed. Antibodies from HIV HEC-immunized macaques still recognized HIV HEC analogs representing the monovalent epitopes of five variable regions of gp120 from subtype B HIV-1 MN, HIV-1 RF and HIV-1 SF2 isolates with an antibody titer greater than 1:500 dilution (Fig. 1B). These results suggest that a broadly reactive, memory antibody response can be detected several years after immunization with HIV HECs. Moreover, lymphocytes from lymph nodes of HIV
HEC-immunized macaques showed $\mathrm{T}$ cell proliferative responses specific to HIV HEC individual components and to the five HIV HEC peptides combined (Fig. 1C). This result also suggest that the memory $\mathrm{T}$ cell response induced by the HIV HECs were well preserved even 8 years after the last immunization.

\section{CONCLUSION}

Most HIV vaccine studies have mainly focused on induction of either B cell or CD8 T cell responses (Virgin and Walker, 2010). Both an antibody response and killing of HIV-infected cells by CD8 T cells would need help from CD4 $\mathrm{T}$ cells to limit HIV infection or reduce HIV replication. A successful preventive or therapeutic HIV vaccine should be able to simultaneously elicit CD4 T helper cells, CD8 cytotoxic T cells and a broadly, high affinity neutralizing antibody response.

In our previous studies, we demonstrated that mice, rabbits and rhesus macaques immunized with HIV-1 HECs developed strong antibody responses specific to the immunogens and broadly neutralizing antibodies that inhibit viral infection of three different HIV-1 strains (Carlos et al., 2000). Our laboratory also reported that lymphocyte proliferative responses in HIV HECimmunized animals were specific to the HIV-1 HEC immunogens and were cross-reactive to their analogs as well as subtype-specific peptides (Carlos et al., 2000). In one of our previous studies, rhesus macaques immunized 
with SIV HECs developed strong, cross-reactive antibody responses to envelope proteins from divergent strains of SIV (Anderson et al., 2005). Moreover, rhesus macaques immunized with SIV HECs and challenged intravenously with SIVmac251, made neutralizing antibodies targeting the challenge strain of virus (Anderson et al., 2005). Our previous studies combined suggest that HIV HECs are strongly immunogenic, eliciting both cellular and humoral immune responses specific to the HIV gp120. These studies also suggest that our HIV HECs are able to induce broadly neutralizing antibodies. Recently, we also reported an immunological comparison between lipidated and nonlipidated multivalent HIV-1 peptides representing gp120 and gag hypervariable regions (Ghunaim et al., 2011).

Although we followed up the HIV HEC-specific antibody response up to (Carlos et al., 2000), we did not determine whether memory responses elicited by HIV HECs could be detected several years after immunization. In the present study, performed more than 8 years after two immunizations were administered in a two-month interval, both macaques received a booster immunization to measure memory immune responses specific to HIV HECs. This was done to specifically determine whether HIV HECs could induce the long-lasting anamnestic immune response required after effective vaccination. Our results showed that macaques immunized with the HIV HEC peptide mixture retained strong antibody-producing B cell memory and lymphoproliferative responses specific to HIV HECs. In addition, this memory antibody response could still recognize antigenic epitopes of gp120 from diverse strains of HIV -1 .

Overall, these results suggest that our multivalent peptide mixtures (HIV HECs), are strong immunogens, eliciting both humoral and cellular immunity that is broadly reactive to epitopes found in divergent strains of HIV-1. In addition, HIV HECs are able to elicit not only early effector immune responses, but also strong, longlasting memory anamnestic immune responses without causing adverse effects in non-human primates. These findings could contribute to the design of a vaccine against HIV and AIDS. We believe that these results are relevant to the likely possibility that individuals will be exposed to pathogenic HIV-1 several years following vaccination. In this case, the initial infection would boost the immune response triggering the memory $\mathrm{B}$ and $\mathrm{T}$ cells to reduce the initial viremia and seeding of mucosal lymphoid organs with HIV-1. This effect could result in an immune response that delays the development of AIDS by keeping the virus burden at a very low level in the lymphoid organs.

\section{REFERENCES}

Anderson, D.E., A. Malley, E. Benjamini, M.B. Gardner and J. Torres, 1994. Hypervariable epitope constructs as a means of accounting for epitope variability. Vaccine, 12: 736-740. DOI: 10.1016/0264-410X(94)90225-9

Anderson, D.E., A. Singapuri, K.H. Kang, D.C. Montefiori and D.J.V. Torres et al., 2005. Timing of retroviral infection influences anamnestic immune response in vaccinated primates. Viral Immunol., 18: 689-694. DOI: 10.1089/vim.2005.18.689

Azizi, A., D.E. Anderson, M. Ghorbani, C. Soare and P. Sandstrom et al., 2008. Induction of broad crosssubtype-specific HIV-1 immune responses by a novel multivalent HIV-1 peptide vaccine in cynomolgus macaques. J. Immunol., 180: 21742186. PMID: 18250424

Carlos, M.P., D.E. Anderson, M.B. Gardner and J.V. Torres, 2000. Immunogenicity of a vaccine preparation representing the variable regions of the HIV type 1 envelope glycoprotein. AIDS Res. Hum. Retroviruses, 16: 153-161. DOI: 10.1089/088922200309494

Gallo, R.C., 2005. The end or the beginning of the drive to an HIV-preventive vaccine: A view from over 20 years. Lancet, 366: 1894-1898. DOI: 10.1016/S0140-6736(05)67395-3

Ghunaim, H., A. Kumar, J. Torres, F. Diaz-Mitoma and A. Azizi et al., 2011. An immunological comparison between lipidated and non-lipidated multivalent HIV-1 peptides representing Gp120 and Gag hypervariable regions. Vaccine, 29: 5950-5958. DOI: 10.1016/j.vaccine.2011.06.047

Kang, K.H., Y. Yamamura, M.P. Carlos, N. Karvelas and I.S. Kim et al., 2010. Synthetic antigens representing the antigenic variation of human hepatitis C virus. Viral Immunol., 23: 497-508. DOI: 10.1089/vim.2010.0043

Kwong, P.D., M.L. Doyle, D.J. Casper, C. Cicala and S.A. Leavitt et al., 2002. HIV-1 evades antibodymediated neutralization through conformational masking of receptor-binding sites. Nature, 420: 678682. DOI: $10.1038 /$ nature 01188

Levy, J.A., 2006. HIV pathogenesis: Knowledge gained after two decades of research. Adv. Dent. Res., 19: 10-16. DOI: 10.1177/154407370601900104

Ndung'u, T. and R.A. Weiss, 2012. On HIV diversity. AIDS, 26: 1255-1260. DOI: $10.1177 / 154407370601900104$ 
Overbaugh, J. and L.M. Rudensey, 1992. Alterations in potential sites for glycosylation predominate during evolution of the simian immunodeficiency virus envelope gene in macaques. J. Virol., 66: 59375948. PMID: 1527847

Reddy, K.J., B. Banapour, D.E. Anderson, S.H. Lee and J.P. Marquez et al., 2004. Induction of immune responses against human papillomaviruses by hypervariable epitope constructs. Immunology, 112: 321-327. DOI: 10.1111/j.1365-2567.2004.01873.x

Saunders, K.O., R.S. Rudicell and G.J. Nabel, 2012. The design and evaluation of HIV-1 vaccines. AIDS, 26: 1293-1302. DOI: 10.1097/QAD.0b013e32835474d2
Shan, M., P.J. Klasse, K. Banerjee, A.K. Dey and S.P.N. Iyer et al., 2007. HIV-1 gp120 mannoses induce immunosuppressive responses from dendritic cells. PLoS, Pathog., 3: e169-e169. DOI: 10.1371/journal.ppat.0030169

Virgin, H.W. and B.D. Walker, 2010. Immunology and the elusive AIDS vaccine. Nature, 464: 224-231. DOI: $10.1038 /$ nature 08898

Wyatt, R., P.D. Kwong, E. Desjardins, R.W. Sweet and J. Robinson et al., 1998. The antigenic structure of the HIV gp120 envelope glycoprotein. Nature, 393: 705-711. DOI: 10.1038/31514 